\title{
Dysfunctional high-density lipoprotein: not only quantity but first of all quality?
}

Aris P. Agouridis ${ }^{1,2}$, Maciej Banach³, Dimitri P. Mikhailidis ${ }^{4}$

\author{
${ }^{1}$ Department of Internal Medicine, Medical School, University of Ioannina, Ioannina, \\ Greece \\ ${ }^{2}$ Department of Clinical Biochemistry (Vascular Disease Prevention Clinics), Royal Free \\ London Foundation Trust, London, UK \\ ${ }^{3}$ Department of Hypertension, Chair of Nephrology and Hypertension, Medical \\ University of Lodz, Lodz, Poland \\ ${ }^{4}$ Department of Clinical Biochemistry (Vascular Disease Prevention Clinics), Royal \\ Free Hospital campus, University College London Medical School, University College \\ London (UCL), London, UK
}

Submitted: 22 January 2015

Accepted: 22 January 2015

Arch Med Sci 2015; 11, 1: 230-231

DOI: 10.5114 /aoms.2015.49816

Copyright (c) 2015 Termedia \& Banach

According to many studies, low levels of high-density lipoprotein cholesterol (HDL-C) are an independent risk factor for coronary artery disease [1]. HDL has several pleiotropic properties apart from reverse cholesterol transport; these include anti-oxidant, anti-inflammatory, anti-infectious, anti-proliferative, antithrombotic and vasodilator actions [2-4].

Randomised controlled trials, prospective and genetic studies, as well as meta-analyses have produced controversial results regarding treating low HDL-C levels $[5,6]$.

The functionality of HDL-C, as well as the role of dysfunctional HDL (dys $\mathrm{HDL}$ ) in the prediction of cardiovascular (CV) risk has generated considerable debate. Therefore, we read with interest the Dodani et al. study [7] which showed that dysHDL was significantly associated $(p=0.0024)$ with common carotid artery intima-media thickness (CCA-IMT) in 129 South Asian immigrants. Chronic inflammation transforms HDL to dys $\mathrm{H}$ DL [4]. However, the authors did not mention any correlation between high sensitivity C-reactive protein levels and the quantity of dysHDL or whether routinely measured HDL-C levels correlated with CCA-IMT and if any such relationship differs from dysHDL.

It is apparent from the above, that not only the quantity, but also the quality of HDL matters (Quantity and Quality; "Q and Q"). As a result, there is a need for new drugs that, apart from raising HDL-C levels, can improve HDL function $[8,9]$. Cholesterol ester transfer protein (CETP) inhibitors (anacetrapib and evacetrapib) seem to be promising [6]. However, there is no class effect, as the development of other CETP inhibitors (torcetrapib and dalcetrapib) was discontinued [6].

It is worth emphasizing that we are at the beginning of dysHDL research. Some issues need to be considered before confirming the potential predictive role of dysHDL [4]. We need an easy and direct diagnostic method for dysHDL evaluation (possibly via measurement of highly specific microRNAs (miRs), as suggested in the DYS-HDL trial), as well as an optimal method for HDL subfraction analysis (to establish if certain subfractions are dysfunctional) $[2,10,11]$.

\author{
Corresponding author: \\ Dimitri P. Mikhailidis MD, \\ FRCP, FRCPath \\ Department of Clinical \\ Biochemistry \\ (Vascular Disease Prevention \\ Clinics) \\ Royal Free Hospital Campus \\ University College London \\ Medical School \\ University College London \\ (UCL) \\ Pond Street, London NW3 \\ 2QG, UK \\ Phone: 02078302258 \\ Fax: 02078302235 \\ E-mail: mikhailidis@aol.com
}


HDL carries specific miRs $[10,11]$. Therefore, dysHDL-miRs, as well as HDL subfraction-miRs, could prove to be novel biomarkers of CV disease [10, 11]. Targeting HDL-miRs might also be a promising basis for drug development $[10,11]$. It is crucial to identify the patients and conditions at the highest risk of dysHDL formation. This population might benefit most from improving HDL "Q and Q" with a potential subsequent reduction in CV disease residual risk [10-13].

\section{Conflict of interest}

This letter was written independently. The authors did not receive financial or professional help with the preparation of the manuscript. DPM has given talks, attended conferences and participated in studies sponsored by Merck, Sharp \& Dohme (MSD) and Genzyme. APA is supported by a grant from the Hellenic Atherosclerosis Society. MB has given talks, attended conferences and participated in studies sponsored by Merck, Sharp \& Dohme (MSD), Abbott, Sanofi and Amgen. He is a member of the Amgen International Advisory Board.

\section{Acknowledgments}

The study was financed by the Polish National Science Centre (OPUS Grant, contract No. DEC2013/09/B/NZ5/02746).

\section{References}

1. Executive Summary of The Third Report of The National Cholesterol Education Program (NCEP) Expert Panel on Detection, Evaluation, And Treatment of High Blood Cholesterol In Adults (Adult Treatment Panel III). JAMA 2001; 285: 2486-97.

2. Rizzo M, Otvos J, Nikolic D, Montalto G, Toth PP, Banach $M$. Subfractions and subpopulations of HDL: an update. Curr Med Chem 2014; 21: 2881-91.

3. Florentin M, Liberopoulos EN, Wierzbicki AS, Mikhailidis DP. Multiple actions of high-density lipoprotein. Curr Opin Cardiol 2008; 23: 370-8.

4. Otocka-Kmiecik A, Mikhailidis DP, Nicholls SJ, Davidson M, Rysz J, Banach M. Dysfunctional HDL: a novel important diagnostic and therapeutic target in cardiovascular disease? Prog Lipid Res 2012; 51: 314-24.

5. Toth PP, Barylski M, Nikolic D, Rizzo M, Montalto G, Banach $M$. Should low high-density lipoprotein cholesterol (HDL-C) be treated? Best Pract Res Clin Endocrinol Metab 2014; 28: 353-68.

6. Florentin M, Kostapanos MS, Kei A, Elisaf M. Emerging drugs for hyperlipidaemia: an update. Expert Opin Emerg Drugs 2014; 19: 471-88.

7. Dodani S, Dong L, Guirgis FW, Reddy ST. Carotid intima media thickness and low high-density lipoprotein (HDL) in South Asian immigrants: could dysfunctional HDL be the missing link? Arch Med Sci 2014; 10: 870-9.

8. Gomaraschi M, Adorni MP, Banach M, Bernini F, Franceschini G, Calabresi L. Effects of established hypolipidemic drugs on HDL concentration, subclass distribution, and function. Handb Exp Pharmacol 2015; 224: 593-615.
9. Dragan S, Serban C, Banach M. Can we change the functionality of HDL cholesterol with nonpharmacological and pharmacological agents? Curr Med Chem 2014; 21 : 2927-46.

10. Banach M, Rysz J, Rizzo M, et al. Investigating dysfunctional-HDL in selected groups of patients at high risk of cardiovascular events (DYS-HDL study) - Protocol and organization. Atherosclerosis 2014; 235: e171-2.

11. Serban C, Muntean D, Mikhailidis DP, Toth PP, Banach M. Dysfunctional HDL: the journey from savior to slayer. Clin Lipidol 2014; 9: 49-59.

12. Kucera M, Oravec S, Hirnerova E, et al. Effect of atorvastatin on low-density lipoprotein subpopulations and comparison between indicators of plasma atherogenicity: a pilot study. Angiology 2014; 65: 794-9.

13. Al-Zakwani I, Sulaiman K, Al-Rasadi K, Mikhailidis DP. Prevalence of low high-density lipoprotein cholesterol (HDL-C) as a marker of residual cardiovascular risk among acute coronary syndrome patients from Oman. Curr Med Res Opin 2011; 27: 879-85. 